\title{
Gains in health utility associated with urinary catheter innovations
}

\author{
Andrei Krassioukov ${ }^{1,2}$ \\ Yasuhiko Igawa ${ }^{3}$ \\ Márcio Augusto Averbeck ${ }^{4}$ \\ Helmut Madersbacher ${ }^{5}$ \\ Andrew J Lloyd ${ }^{6}$ \\ Mette Bøgelund ${ }^{7}$ \\ Nikesh Thiruchelvam ${ }^{8}$ \\ 'University of British Columbia, \\ International Collaboration On \\ Repair Discoveries (ICORD), \\ Vancouver, British Columbia, Canada; \\ ${ }^{2}$ Division of Physical Medicine and \\ Rehabilitation, Department of \\ Medicine, G.F. Strong Rehabilitation \\ Centre, Vancouver, British Columbia, \\ Canada; ${ }^{3}$ Department of Continence \\ Medicine, The University of Tokyo, \\ Graduate School of Medicine, Tokyo, \\ Japan; ${ }^{4}$ Head of Neuro-Urology \\ Unit, Moinhos de Vento Hospital, \\ Porto Alegre, Brazil; ${ }^{5}$ Department \\ of Neurology, Medical University \\ Innsbruck, Innsbruck, Austria; \\ ${ }^{6}$ Bladon Associates Ltd., Oxford, \\ UK; ${ }^{7}$ Incentive Partners ApS, Holte, \\ Denmark; ${ }^{8}$ Department of Urology, \\ Cambridge University Hospitals NHS \\ Trust, Cambridge, UK
}

Correspondence: Andrei Krassioukov University of British Columbia, International Collaboration On Repair Discoveries (ICORD), 818 West 10th Avenue, Vancouver, BC V5Z IM9,

Canada

$\mathrm{Tel}+\mathrm{I} 6046758819$

Fax +I 6046758820

Email krassioukov@icord.org
This article was published in the following Dove Press journal: Medical Devices: Evidence and Research

\begin{abstract}
Purpose: To estimate gains in health utility for two different catheter features and a support service related to urinary catheters used for intermittent catheterization.
\end{abstract}

Patients and methods: Two internet-based time trade-off (TTO) surveys were undertaken to value vignettes describing two innovative catheter features and a support service. The first TTO survey "Size and Service" included catheters with compact design and the availability of a support service for users; the second TTO survey "Phthalates" included avoidance of potentially harmful phthalates in the material of the catheters. All participants were from the UK; they traded health states against a time horizon that matched their total life expectancy. Sensitivity analyses were done to estimate the impact of extreme values on disutilities.

Results: The participants $(\mathrm{n}=890)$ estimated the incremental value of 0.031 (95\% CI: 0.024 0.039), 0.009 (95\% CI: 0.003-0.015), and 0.037 (95\% CI: 0.027-0.046), respectively, for catheters with compact design, availability of support service, and catheters not containing phthalates. Conclusions: Participants valued all three improvements in catheter design. To capture the impact of such design improvements on quality of life and utilities, vignette-based approaches can be a useful supplement to the conventional, generic tools.

Keywords: intermittent catheterization, time trade-off, vignette-based approach, health-related quality of life, QALY

\section{Introduction}

Conditions like traumatic spinal cord injuries and spina bifida leave patients reliant on urinary catheters for bladder emptying. ${ }^{1}$ It is generally accepted by organizations such as the National Institute for Health and Care Excellence (NICE) and the European Association of Urology that intermittent catheterization is recommended over use of indwelling catheters, since it is associated with considerably less urinary tract infections (UTIs) and complications..$^{2-4}$

The desire to simplify the practical aspects of the catheterization procedure as well as user preferences has driven innovations in both catheter design and catheter composition (manufacturing). By design, compact catheters are smaller than the conventional ones and are therefore considered to be more discreet and convenient, as measured by the validated Intermittent Self-Catheterization Questionnaire (ISC-Q). ${ }^{5,6}$ An innovation in catheter composition includes avoidance of the chemical softeners phthalates, which are suspected of having a negative impact on human health. ${ }^{7-9}$

In addition to these technical improvements in catheter design and materials, there is also an improved understanding of the role of better patient support and 
education to minimize catheter-associated problems. Best practice regarding hygiene, frequency of use, and other aspects of catheter use have caused a decrease in the frequency of UTIs. ${ }^{3}$ Therefore, the provision of better support for users could have an important effect on catheter-related outcomes. ${ }^{10}$

In many health care systems, decision makers have a formal remit to maximize value. Typically, this means preferring treatments that improve survival and/or quality of life (QoL). ${ }^{11}$ Cost utility analyses are used to estimate the efficiency of new health technologies in terms of their cost and benefits, expressed as length and QoL. The conventional decision-making framework does not normally consider improvements to the process of receiving care, unless these improvements have a measurable benefit in terms of effectiveness, such as improving the QoL. ${ }^{12}$ The issue of the potential risk of phthalates to long-term health is also complex to consider in a model, because of uncertainties in the data; this issue is perhaps best represented in terms of the value that society places on their avoidance. Since support services, product discreetness/convenience, and the issue of phthalates may be of value to patients and to the general public, these features should also be considered within a decision framework. There is a lot of debate in the literature regarding what should be considered by health technology assessment (HTA) decision makers. ${ }^{13}$ For individuals needing to use intermittent catheters (IC), bladder management is an essential part of health care, and any valid improvement in their bladder management should therefore be considered by decision makers.

One way to assess this type of benefit in catheter design and bladder management is the usage of patient preference surveys, such as discrete choice experiments. ${ }^{14,15}$ However, the results from discrete choice experiments cannot be used to express patient benefits in cost-utility analyses. ${ }^{12}$ As an alternative, time trade-off (TTO) surveys could be used to estimate the value of catheter innovations. The TTO method explores value by understanding the extent to which people are willing to trade duration of survival. TTO methods are commonly used to assess QoL, but have previously been used to explore the value of issues related to the process of care, for example, in the studies by Ridderstråle et $\mathrm{al}^{16}$ and Brennan and Dixon. ${ }^{12}$

The present study was designed to explore the value of catheter innovations, using the TTO method to estimate disutilities associated with three different catheter product features: access to a patient support service, the use of catheters that do not contain phthalates, and the usage of catheters that are of a compact design.

\section{Material and methods}

We conducted two separate TTO surveys to explore different features of catheters, so that each participant completed only a smaller number of assessments. The sample size in the present study was larger than similar interview-based vignette studies that have been reported in the past, so that the sensitivity of the study was sufficiently high. ${ }^{17,18}$ In addition, the trading period in the TTO exercise was not limited to 10 years as is often the case ${ }^{19}$ but was extended to each participant's remaining life expectancy.

\section{Health state vignette development}

We developed a series of health-related vignettes for estimating the value or utility associated with improvements in catheter design. Each participant was presented with a general state that described the impact of relying on IC use for bladder management. The participants were asked to imagine that they were reliant on IC for their bladder management. They were not given any other information such as their etiology or any other functional loss (Figure 1).

Subsequently, the patients entered one of the two TTO surveys. Each respondent answered only one of the surveys. The first TTO survey assessed "Size and Service", and included three health states based on the size of the catheter (regular or compact) and on the availability of a specialized web-based or phone-based support service for resolving issues related to the usage of catheters. The second TTO survey assessed the value of avoiding phthalates, and included two health states based on whether the catheter was phthalate-free or not. The health states are summarized in Table 1. In addition, a detailed description about the nature of supportive services was given for the first survey, and basic information about phthalates was given for the second survey (Table 2).

\section{Participants and procedures}

Both TTO surveys were conducted in 2017 and recruited a representative sample of the adult UK population. Respondents were recruited from the general population since several HTA guidelines recommend that health state utilities represent the values of the general population rather than users or patients with the specific condition. ${ }^{20-22}$ Both TTO surveys were web based and used the members of an existing panel of research participants, who were compensated for their time (compensation was $<£ 1$ ). 
"Imagine that you have a disease that makes you unable to urinate/pee naturally.

In order to empty your bladder, you need to use a catheter every 4-6 hours, or four to six times per day, following your normal toilet pattern.

You will not be able to pee without a catheter and you will most likely not feel the sensation of urge to pee.

A catheter is illustrated below. It is a thin, flexible, hollow tube of approximately 16 inches (male)/8 in (female) that allows the urine to drain out from the bladder.

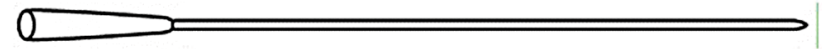

You insert the catheter through your penis (male) /above the vaginal opening (female) to reach the bladder until urine flows.

The use of catheters will most likely increase the risk of having urinary tract infections. This will potentially increase the risk of severe infections in the kidneys.

When you get a urinary tract infection you will need antibiotic treatment, and for a couple of days, you will most likely be sick with fever and be unable to perform your daily routines.

Imagine that you have to live with this for the rest of your life."

Figure I An example vignette from valuation exercise.

Table I Health states and levels for "Size and service" and "Phthalates"

\begin{tabular}{|c|c|c|}
\hline Size and service & Male & Female \\
\hline $\begin{array}{l}\text { HS I: Regular-sized } \\
\text { catheter }\end{array}$ & $\begin{array}{l}\text { - } 47.5-\mathrm{cm} \text {-long catheter packing } \\
\text { - Not discreet } \\
\text { - Somewhat easy to carry four to six catheters around } \\
\text { every day }\end{array}$ & $\begin{array}{l}\text { - 19-cm-long catheter packaging } \\
\text { - Not discreet } \\
\text { - Somewhat easy to carry four to six catheters around } \\
\text { every day }\end{array}$ \\
\hline $\begin{array}{l}\text { HS 2: Regular-sized } \\
\text { catheter and service }\end{array}$ & $\begin{array}{l}\text { - } 47.5-\mathrm{cm} \text {-long catheter packaging } \\
\text { - Not discreet } \\
\text { - Somewhat easy to carry four to six catheters around } \\
\text { every day } \\
\text { - Access to a specialized advisory through phone and } \\
\text { website }\end{array}$ & $\begin{array}{l}\text { - 19-cm-long catheter packaging } \\
\text { - Not discreet } \\
\text { - Somewhat easy to carry four to six catheters around } \\
\text { every day } \\
\text { - Access to a specialized advisory through phone and } \\
\text { website }\end{array}$ \\
\hline $\begin{array}{l}\text { HS 3: Compact-sized } \\
\text { catheter }\end{array}$ & $\begin{array}{l}\text { - } 27.5 \text {-cm-long catheter packaging } \\
\text { - Discreet } \\
\text { - Very easy to carry four to six catheters around every day }\end{array}$ & $\begin{array}{l}\text { - II-cm-long catheter packaging } \\
\text { - Discreet } \\
\text { - Very easy to carry four to six catheters around every day }\end{array}$ \\
\hline Phthalates & & \\
\hline $\begin{array}{l}\text { HS I: Phthalate } \\
\text { catheter }\end{array}$ & A plastic material that contains phthalates & \\
\hline $\begin{array}{l}\text { HS 2: Phthalate-free } \\
\text { catheter }\end{array}$ & A plastic material that is free of phthalates & \\
\hline
\end{tabular}

Abbreviation: $\mathrm{HS}$, health state.

Table 2 Definitions and description of phthalates and additional services

Phthalates Phthalates are a group of chemical softeners often used in plastic materials to make them soft and flexible, for example, rain clothes, shower curtains, and plastic boxes. Phthalates are suspected of having a negative impact on human health by interfering with the hormone and immune system and by causing reproductive and developmental disorders. Phthalates are also suspected of increasing the risk of some types of cancer. There is currently inconclusive evidence on the exact impact on human health, but as a precautionary measure authorities in several countries have banned phthalates in children's toys. Also, The European Chemical Agency has included phthalates on its list of substances of very high concern, which should be removed from consumer products for human contact.

Additional When you use a catheter, you can always contact your general practitioner or health clinic, if you have questions or concerns related service to the product. When you use certain types of catheters you will have additional access to a specialized service that offers advice and guidance on how to cope in your daily life with a catheter. You get access to both a direct phone line to trained staff and a designated website with tips and tricks. 
All respondents provided demographic (age, gender, employment, household) and socioeconomic characteristics (income), as well as details regarding experience with IC. The web surveys incorporated a 10 -second delay before participants could proceed to the next page, to prevent the respondents from answering without reading the questions. Warm-up questions were included to familiarize respondents with the methodology and to avoid errors in answering questions. A test question was included to identify irrational responders or nontraders by asking respondents if they preferred to live a longer time in full health or a shorter life with daily use of IC. Any respondents who preferred the shorter life (with worse QoL) were excluded from the dataset. In the main survey, the time horizon in the TTO task was chosen to match the expected survival of each participant, based on life expectancy data. To make the trade-offs as realistic as possible, the time horizons used were based on each respondent's projected life expectancy, obtained using the country, age, and sex of the respondent at the time of the study, and the most recent World Health Organization life tables. ${ }^{23}$

A pilot study was conducted in the UK with 20 respondents. Based on feedback from the pilot study, the description of the phthalates issue was revised so that it was better understood by the respondents. It was also decided to undertake this research in two separate surveys which separately assessed "Size and Service" and "Phthalates".

\section{Statistical analyses}

We used $\mathrm{SAS}^{\circledR}$ version 9.4 software for statistical analyses. Utilities were calculated for each health state and the incremental value for each health state was estimated as the arithmetic difference between two states. Bootstrapping with 10,000 iterations was utilized to estimate CI for the mean utility values. Base results were based on a $5 \%$ extreme value outlier's exclusion. A sensitivity analysis was carried out based on $100 \%, 99 \%$, and $0 \%$ inclusion levels.

\section{Ethical statement}

This study was carried out within the codes of conduct of the Market Research Society and in compliance with the applicable European Society for Opinion and Marketing Research (ESOMAR) guidelines. The respondents were asked to complete an informed consent form before being able to access the survey. Ethical review board approval was not required for this type of study in the UK, since the study was not a clinical trial, and since no human or biological samples or identifiable personal information was collected.

\section{Results}

A total of 890 respondents completed the survey (455 completed the "Size and Service" survey, and 435 completed the "Phthalates" survey). A total of 9\% (45) and 11\% (54) respondents failed the test questions in the "Size and Service" survey and "Phthalate" survey, respectively, and hence were excluded from the survey.

The demographic and economic details of the respondents were similar in both the surveys (Table 3 ). Use of the compact-sized catheter was associated with a utility gain of 0.031 (95\% CI: 0.024-0.039) when compared with the use of a regular-sized catheter. Access to a specialized service for catheter-related problems was associated with an incremental utility gain of 0.009 (95\% CI: $0.003-0.015)$. A catheter without phthalates was associated with a utility gain of 0.037 (95\% CI: 0.027-0.046) compared with a catheter that contained phthalates.

The sensitivity analysis showing the impact of different cut-off limits for extreme values on the utility gains is depicted in Figure 2. The 95\% cut-off was used as the base case, meaning that the $5 \%$ of values that are at the extreme

Table 3 Demographic and socioeconomic characteristics of the respondents used for the analysis

\begin{tabular}{|c|c|c|}
\hline Features & $\begin{array}{l}\text { Size and } \\
\text { service }\end{array}$ & Phthalates \\
\hline $\mathrm{N}$ & 455 & 435 \\
\hline Male:Female & $\mathrm{I}: \mathrm{I}$ & $\mathrm{I}: \mathrm{I}$ \\
\hline Age in years, mean $\pm S D$ & $44.1 \pm 19.9$ & $44.5 \pm 15.4$ \\
\hline \multicolumn{3}{|l|}{ Age-wise distribution } \\
\hline - $<30$ & $109(24 \%)$ & $112(26 \%)$ \\
\hline - 30-39 & $70(15 \%)$ & $60(14 \%)$ \\
\hline - $40-49$ & III (24\%) & $81(19 \%)$ \\
\hline - 50-59 & $81(18 \%)$ & $97(22 \%)$ \\
\hline - $60-69$ & $72(16 \%)$ & $82(19 \%)$ \\
\hline - $>70$ & $12(3 \%)$ & $3(1 \%)$ \\
\hline In paid employment & $25 \mathrm{I}(55 \%)$ & $253(58 \%)$ \\
\hline \multicolumn{3}{|l|}{ Living situation } \\
\hline - One adult without children & $88(19 \%)$ & $89(20 \%)$ \\
\hline - One adult with children & $22(5 \%)$ & $19(4 \%)$ \\
\hline - Two or more adults with children & 202 (44\%) & $200(46 \%)$ \\
\hline - Two or more adults without children & $143(31 \%)$ & 127 (29\%) \\
\hline \multicolumn{3}{|l|}{ Income per month } \\
\hline - $£ 0-£ 999$ & $67(15 \%)$ & $54(12 \%)$ \\
\hline - $f 1000-f 1999$ & $120(26 \%)$ & $123(28 \%)$ \\
\hline - $£ 2000-£ 3999$ & 157 (35\%) & $138(32 \%)$ \\
\hline - $£ 4000-£ 5999$ & $42(9 \%)$ & $38(9 \%)$ \\
\hline - $£ 6000-£ 8999$ & $17(4 \%)$ & $12(3 \%)$ \\
\hline - $\geq £ 9000$ & $18(4 \%)$ & $29(7 \%)$ \\
\hline - Do not wish to answer & $34(7 \%)$ & $41(9 \%)$ \\
\hline \multicolumn{3}{|l|}{ Experience with catheters } \\
\hline - Use catheter on a daily basis & $13(3 \%)$ & $11(3 \%)$ \\
\hline - Have previously used a catheter & $54(12 \%)$ & $57(13 \%)$ \\
\hline
\end{tabular}




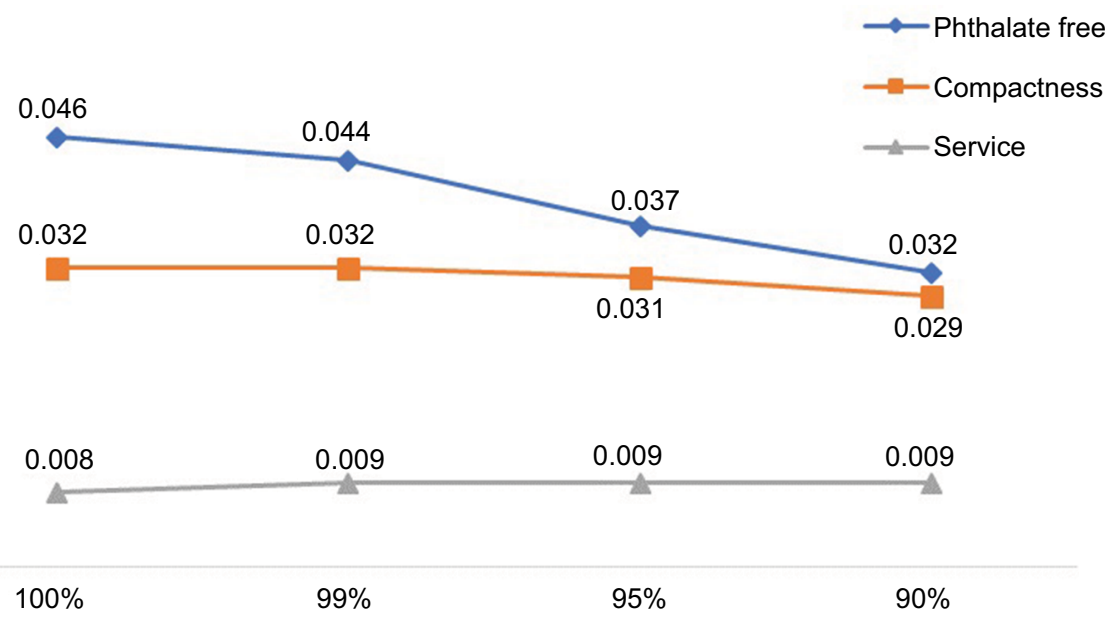

Figure 2 Sensitivity analysis of utility estimates for compact design, provision of support service, and use of phthalates.

Notes: The graph shows the effect on disutility estimates of excluding outliers or extreme values: no exclusion (100\%), $1 \%$ exclusion ( $99 \%), 5 \%$ exclusion ( $95 \%$, base results), and $10 \%$ exclusion $(90 \%)$.

ends of the range ( $2.5 \%$ in each end) were excluded from the analysis. The utility gain for phthalate-free catheters was quite sensitive to the exclusion of extreme values. The disutility of catheters containing phthalates was greatest when all participants were included, and this declined as more of the extreme values were excluded. This suggests that a small proportion of participants placed quite a large value on the avoidance of phthalates. In contrast, the provision of a catheter support service had a consistent effect on utility regardless of the removal of extreme values. This suggests that the support service was valued quite consistently by the sample.

\section{Discussion}

The present study uses the TTO method to estimate values for aspects of bladder management which fall outside of the health dimensions measured by the EQ-5D. It is interesting to note that the study participants were willing to trade against catheter innovations and the provision of a support service, despite certain limitations in the study design (as discussed below). The study suggests that aspects such as compact-sized catheters, availability of support services, and avoidance of phthalates, are of value to society and therefore decision makers should also consider these innovations to fully understand the benefit of catheter innovation. The results show that the respondents value catheters without phthalates because these chemicals have a potentially harmful effect on human health. Little research has been done on the preference of IC users regarding phthalates, but the results are in line with a reported increased demand for phthalate-free catheters and with government recommendations to seek alternatives to phthalates in medical devices, where possible., ${ }^{9,24}$ The significant utility benefit from compact, discreet catheters is consistent with the results from a randomized trial comparing compact vs standard-sized catheters, which showed a $28 \%$ increase in catheter-related QoL as measured by the validated Intermittent Self-Catheterization Questionnaire (ISC-Q). The study also showed that $63 \%$ of IC users preferred the compact catheter. ${ }^{5}$ The specialized support service was also found to have a significant utility benefit, but was valued lowest among the three attributes. The relatively low utility benefit contrasts with research showing that IC poses a challenge to the daily life for many users and that specialized and ongoing support/follow-up is recommended to increase patients' $\mathrm{QoL}$ and adherence to IC as a treatment regime. ${ }^{25,26}$ More research is needed on the impact of specialized IC support services to understand if the current results validly represent the benefits of such support services.

The use of quality-adjusted life years (QALYs) as the standard metric of benefit in cost-utility analyses in health care has stood the test of time. Standard measures such as EQ-5D focus on certain dimensions of health such as mobility, self-care, usual activities, pain, discomfort, and psychological impact (depression or anxiety) for measuring QALYs. ${ }^{27}$ However, a health technology may help people to manage their medical conditions without necessarily impacting these concepts, and EQ-5D may therefore not be suitable for measuring the specific impairment of health-related quality of life (HRQoL) seen in patients dependent on intermittent catheterization for bladder management. For these reasons, EQ-5D is not sufficient for identifying the specific benefits that innovations in bladder management technologies may provide to the users. ${ }^{15,28,29}$ 
Technological improvements in catheter design may reduce the burden of bladder management, but they also render the device more expensive. For the healthcare providers to implement new technologies, there should be evidence supporting the gain in health utilities by the usage of the more expensive devices over the less expensive ones. Since EQ-5D and other related standardized tools are not well suited to assess the benefits of catheter innovation, alternate methods to assess these benefits are required. The vignette methodology using the TTO survey applied in this study is a reasonable alternative approach. The present study has identified a relatively small but significant gain in utility associated with compact catheter designs, the provision of a support service, and the avoidance of phthalates in catheters. The utility values have face validity, and the levels are comparable to other process-related utilities found in previous studies. ${ }^{12,18}$

The present study has some limitations. The data in this study were obtained via an online survey rather than a faceto-face interview, to facilitate sampling of data from much larger pools of participants. However, this method also has the inherent weakness that the participants might not have had a complete understanding of the different health states, without an interviewer being present to explain the nuances involved. ${ }^{30}$ Secondly, the health state vignettes that we constructed were simple and particularly focused on catheterization, without touching on other aspects of HRQoL. The simplified health states deliberately avoided any description of the burden of needing intermittent catheterization for patients. This may have drawn the focus of the participants to only these issues, thereby potentially exaggerating their importance. It is also not possible to know what exact assumptions were made by the participants regarding other aspects of health, such as physical functioning and mental health that were not mentioned in the descriptions.

We argue that the HRQoL tools available for estimating QALYs may not be adequate for assessing the full impact of innovations in IC for bladder management. Improving the care of these patients may produce outcomes such as reduced dependence, improved well-being, enhanced life satisfaction, and improved social relationships. These concepts are difficult to measure using a tool such as the EQ-5D in the standard QALY approach. For this reason, researchers have also considered alternative outcome measures for measuring care such as the Adult Social Care Outcomes Toolkit (ASCOT). ${ }^{31}$ Others have recommended different measurement concepts such as a focus on "well-being" rather than QoL. ${ }^{32}$ Further, there have been instances in the recent past where decision makers have recognized the importance of considering alternatives to the EQ-5D for assessing care. In the UK, NICE, the Medical Research Council and the EuroQol Group have jointly funded a research initiative to develop a measure that goes beyond the basic QALY to incorporate aspects of wellbeing. ${ }^{33}$ This new e-QALY measure and the ASCOT both are expected to provide a single preference-weighted index score so that the value of an improvement or deterioration in care can be valued. Future evaluations of bladder management done by incorporating the measurement of well-being alongside that of the health benefits could further throw light on this aspect of outcome measurement.

\section{Conclusion}

This TTO-based survey found significant health utility gains in relation to a catheter support service, compactly sized catheters, and avoidance of phthalates in the catheter material. The results from this study can be used to support a cost-utility analysis. The approach of using vignettes to value catheter innovations that were used in this study can be used as a supplement to generic measures, such as EQ-5D, when these are found to be insufficient due to the nature of the concepts being assessed.

\section{Acknowledgments}

The authors would like to thank Marksman Healthcare Communications for editorial services and Jeppe Sørensen from Coloplast $\mathrm{A} / \mathrm{S}$ for contributing with interpretation of data and a scientific review of the manuscript. Coloplast $\mathrm{A} / \mathrm{S}$ provided research funding for Incentive Partners Aps for conducting the survey and for Andrew Lloyd for expert advice and medical writing. Marksman Healthcare Communications received funding from Coloplast $\mathrm{A} / \mathrm{S}$ for editorial services.

\section{Author contributions}

All the authors contributed to the survey design, interpretation of the results, and reporting of the study and the underlying work. All authors contributed toward data analysis, drafting and revising the paper and agree to be accountable for all aspects of the work. The authors take full responsibility for the content of this manuscript.

\section{Disclosure}

AK, MAA, NT, and YI occasionally provide expert knowledge to advisory boards at Coloplast $\mathrm{A} / \mathrm{S}$. MB is employed by Incentive Partners Aps. The authors report no other conflicts of interest in this work

\section{References}

1. Pilloni S, Krhut J, Mair D, Madersbacher H, Kessler TM. Intermittent catheterisation in older people: a valuable alternative to indwelling catheter? Age Aging. 2005;34(1):57-60. 
2. Groen J, Pannek J, Castro Diaz D. Summary of European Association of Urology (EAU) Guidelines on Neuro-Urology. Eur Urol. 2016;69(2):324-333.

3. National Clinical Guideline Centre. Infection: Prevention and Control of Healthcare-Associated Infections in Primary and Community Care: Partial Update of NICE Clinical Guideline 2. NICE Clinical Guidelines; No. 139. London: Royal College of Physicians; 2012.

4. National Institute for Health and Clinical Excellence (NICE). Healthcare-Associated Infections: Prevention and Control in Primary and Community Care. 2012. Available from: https://www.nice.org.uk/ guidance/CG139. Accessed May 1, 2018.

5. Chartier-Kastler E, Amarenco G, Lindbo L, et al. A prospective, randomized, crossover, multicenter study comparing quality of life using compact versus standard catheters for intermittent self-catheterization. J Urol. 2013;190(3):942-947.

6. Pinder B, Lloyd AJ. Development of a patient-reported outcome measure focusing on intermittent catheter-related quality of life. Int $J$ Phys Med Rehabil. 2013;1:155.

7. Mallow EB, Fox MA. Phthalates and critically ill neonates: device-related exposures and non-endocrine toxic risks. J Perinatol. 2014;34(12):892-897.

8. Testai E, Hartemann P, Rastogi SC, et al; Ms Scientific Committee SCENIHR. The safety of medical devices containing DEHP plasticized PVC or other plasticizers on neonates and other groups possibly at risk (2015 update). Regul Toxicol Pharmacol. 2016;76:209-210.

9. Johansson K, Greis G, Johansson B, et al. Evaluation of a new PVCfree catheter material for intermittent catheterization: a prospective, randomized, crossover study. Scand J Urol. 2013;47(1):33-37.

10. Oh SJ, Ku JH, Lim SH, Jeon, HG, Son H. Effect of a "centralized intensive education system" for clean intermittent self-catheterization in patients with voiding dysfunction who start catheterization for the first time. Int J Urol. 2006;13(7):905-909.

11. Drummond M, Sculpher M, Torrance G, O'Brian B, Stoddart G. Methods for Economic Evaluation of Health Care Programs. Oxford: Oxford University Press; 2005.

12. Brennan VK, Dixon S. Incorporating process utility into quality adjusted life years: a systematic review of empirical studies. Pharmacoeconomics. 2013;31(8):677-691.

13. Daniels N, Porteny T, Urritia J. Expanded HTA: enhancing fairness and legitimacy. Int J Health Policy Manag. 2016;5(1):1-3.

14. Wilkinson G, Drummond M. Alternative approaches for assessing the socioeconomic benefits of medical devices: a systematic review. Expert Rev Med Devices. 2015;12(5):629-648.

15. Pinder B, Lloyd A, Nafees B, Elkin EP, Marley J. Patient preferences and willingness to pay for innovations in intermittent self-catheters. Patient Prefer Adherence. 2015;9(9):381-388.

16. Ridderstråle M, Evans LM, Jensen HH, et al. Estimating the impact of changes in $\mathrm{HbAlc}$, body weight and insulin injection regimen on health related quality-of-life: a time trade off study. Health Qual Life Outcomes. 2016;14:13.

17. Swinburn P, Shingler S, Acaster S, Lloyd A, Bonthapally V. Health utilities in relation to treatment response and adverse events in relapsed refractory Hodgkin lymphoma and systemic anaplastic large cell lymphoma. Leuk Lymphoma. 2015;56(6):1839-1845.

18. Matza LS, Stewart KD, Davies EW, Hellmund R, Polonsky WH, Kerr D. Health state utilities associated with glucose monitoring devices. Value Health. 2017;20(3):507-511.
19. Matza LS, Boye KS, Feeny DH, et al. The time horizon matters: results of an exploratory study varying the timeframe in time trade-off and standard gamble utility elicitation. Eur J Health Econ. 2016;17(8): 979-990.

20. National Institute for Health and Clinical Excellence (NICE). Guide to the Methods of Technology Appraisal 2013. 2013. Available from: www.nice.org.uk/process/pmg9. Accessed January 18, 2018.

21. CADTH. Guidelines for the Economic Evaluation of Health Technologies: Canada. 2017; 4th Edition. Available from: https://www. cadth.ca/sites/default/files/pdf/guidelines_for_the_economic_evaluation_of_health_technologies_canada_4th_ed.pdf. Accessed May 22, 2017.

22. Pharmaceutical Benefits Advisory Committee. Guidelines for Preparing A Submission to the Pharmaceutical Benefits Advisory Committee (PBAC Guidelines), Version 5.0. 2016. Available from: https://pbac. pbs.gov.au/content/information/files/pbac-guidelines-version-5.pdf Accessed January 18, 2018.

23. WHO. Life Tables for WHO member states. 2014. Available from: http:// www.who.int/gho/mortality_burden_disease/life_tables/life_tables/en/. Accessed October 1, 2017.

24. Agency MaHPR. DEHP phthalates in medical devices; 2015. Available from: https://www.gov.uk/government/publications/dehp-phthalates-inmedical-devices/dehp-phthalates-in-medical-devices. Accessed May 1, 2018.

25. Woodward S, Rew M. Patients' quality of life and clean intermittent self-catheterization. Br J Nurs. 2003;12(18):1066-1074.

26. Vahr S, Cobussen-Boekhorst H, Eikenboom J, et al. Evidence Based Guidelines for Best Practice in Urological Health Care. Catheterisation: Urethral Intermittent in Adults. Dilatation, Urethral Intermittent in Adults. Arnhem: Drukkerij Gelderland; 2013.

27. Brazier J, Ratcliffe J, Saloman J, Tsuchiya A. Measuring and Valuing Health Benefits for Economic Evaluation. Oxford: Oxford University Press; 2016.

28. Haywood KL, Garratt AM, Lall R, Smith JF, Lamb SE. EuroQol EQ-5D and condition-specific measures of health outcome in women with urinary incontinence: reliability, validity and responsiveness. Qual Life Res. 2008;17(3):475-483.

29. Lesen E, Bjorholt I, Ingelgard A, Olson FJ. Exploration and preferential ranking of patient benefits of medical devices: a new and generic instrument for health economic assessments. Int J Technol Assess Health Care. 2017;33(4):463-471.

30. Attema AE, Edelaar-Peeters Y, Versteegh MM, Stolk EA. Time trade-off: one methodology, different methods. Eur J Health Econ. 2013;14(Suppl 1):S53-S64.

31. Netten A, Burge P, Malley J, et al. Outcomes of social care for adults: developing a preference-weighted measure. Health Technol Assess. 2012;16(16):1-166.

32. Peasgood T, Brazier J, Mukuria C, Rowen D. A Conceptual Comparison of Well-Being Measures Used in the UK. Sheffield: Policy Research Unit in Economic Evaluation of Health and Care Interventions (EEPRU); 2014.

33. "EuroQol Research Foundation. "Extending the QALY" project collaboration with NICE. Published Jun 2017. Available from: https://euroqol. org/extending-the-qaly-project-collaboration-with-nice/. Accessed January 18, 2018.

\section{Medical Devices: Evidence and Research}

\section{Publish your work in this journal}

Medical Devices: Evidence and Research is an international, peerreviewed, open access journal that focuses on the evidence, technology, research, and expert opinion supporting the use and application of medical devices in the diagnosis, monitoring, treatment and management of clinical conditions and physiological processes. The identification of novel devices and optimal use of existing devices which will lead to improved clinical outcomes and more effective patient management and safety is a key feature. The manuscript management system is completely online and includes a quick and fair peer-review system. Visit http://www. dovepress.com/testimonials.php to read real quotes from authors. 